\title{
Novel LC-MS/MS method for the quantification of silodosin and its metabolites in human plasma
}

\author{
Priyanka A Shah ${ }^{1}$ \& Pranav S Shrivastav*,2 \\ ${ }^{1}$ DST-INSPIRE Senior Research Fellow, Department of Chemistry, School of Sciences, Gujarat University, Ahmedabad 380009, \\ Gujarat, India \\ 2 Professor of Chemistry, Department of Chemistry, School of Sciences, Gujarat University, Ahmedabad 380009, Gujarat, India \\ * Author for correspondence: Tel.: +91 79 26300969; Fax: +91 79 26308545; pranav_shrivastav@yahoo.com
}

First draft submitted: 4 November 2017; Accepted for publication: 8 November 2017; Published online: 13 December 2017

Keywords: $\alpha_{1}$-adrenoceptor antagonist $\bullet$ benign prostatic hyperplasia $\bullet$ bioanalytical method development $\bullet$ combination therapy • design of experiment $\bullet$ KMD-3213G • KMD-3293 • LC-MS/MS • lower urinary tract symptoms - Silodosin

Benign prostatic hyperplasia (BPH) is a medical condition caused by the enlargement of the prostate gland as a result of hyperplasia of both the glandular epithelial and stromal cell compartments [1]. BPH has become a major cause for the increasing number of lower urinary tract symptoms (LUTS) in men, such as frequent urination, a weak urine stream and dribbling of urine, which can all worsen a patient's quality of life. This disease generally appears at the age of 50 or above in males and is comorbid with hypertension and erectile dysfunction. Moreover, the demographics of the population across the world indicate that aging is one of the most prominent causes of such health issues. To study the symptoms associated with $\mathrm{BPH}$ and bladder outlet obstruction, it is essential to have innovative strategies that can withstand the test of changing times. In response to this need, several safe and effective medical treatment options have been introduced to alleviate the symptoms at an early stage to lower the disease progression rate.

Over the last two decades, $\alpha$-adrenoceptor $(\alpha-\mathrm{AR})$ antagonists have become the first-line treatment therapy for men with LUTS, suggestive of bladder outlet obstruction caused by BPH. Initially, phenoxybenzamine, a nonselective first generation $\alpha_{1}$ - and $\alpha_{2}$-AR antagonist was used to treat LUTS. However, it received limited success because of its associated orthostatic and dizziness-related side effects on account of its poor tolerability. In October 2008, the US FDA approved silodosin as the second, third-generation $\alpha_{1 \mathrm{~A}}$ selective antagonist after tamsulosin for the management of BPH. It has demonstrated significant effectiveness in improving the storage/irritating and voiding/obstructive symptoms compared with a placebo [2]. Furthermore, in a urodynamic study, silodosin assisted in improving detrusor overactivity and in reducing the grade of obstruction [3]. Silodosin is highly selective for the $\alpha_{1 \mathrm{~A}}-\mathrm{AR}$, with an affinity that is 583- and 55.5-fold higher than that for the $\alpha_{1 \mathrm{~B}}$ - and $\alpha_{1 \mathrm{D}}$-ARs, respectively [3,4]. Compared to the currently available $\alpha_{1}$-AR antagonists, silodosin is 1400 -times more specific in terms of $\alpha_{1 A^{-}}$ AR versus $\alpha_{1 B}-A R$ binding than doxazosin and terazosin and 40-times greater compared with tamsulosin. Such increased selectivity for $\alpha_{1 \mathrm{~A}}-\mathrm{AR}$ is envisaged to provide additional relief from LUTS in patients with BPH without increasing the rate of serious adverse cardiovascular events [5]. In this editorial, we evaluate the capabilities and scope of the existing bioanalytical methods and propose a novel fit-for-purpose liquid chromatography-tandem mass spectroscopy (LC-MS/MS) method in human plasma that can meet the current requirements for the analysis of silodosin and its metabolites.

\section{Bioanalytical platforms for silodosin: past, present \& future}

Since its approval in 2008, there have been very few bioanalytical methods developed for the quantitation of silodosin [6-8]. LC-MS/MS so far has been the preferred technique for analysis; however, a complete account of bioanalytical method development incorporating sample extraction, chromatographic separation and mass spectrometric detection is currently limited. The validated LC-MS/MS method reported by Zhao et al. [6] allowed 
the quantification of silodosin in human plasma. Here, the LLOQ of silodosin was $0.50 \mathrm{ng} / \mathrm{ml}$ using $500 \mu \mathrm{l}$ plasma sample. After sample preparation from liquid-liquid extraction with methyl tert-butyl ether, silodosin was analyzed on Zorbax C8 column. Electrospray ionization was performed in the positive mode due to the basic nature of silodosin, and the developed method was further applied to a pharmacokinetic study in two healthy subjects. Metabolism of silodosin via the glucuronidation pathway generates the major silodosin $\beta$-D-glucuronide metabolite designated as KMD-3213G, which has demonstrated significant pharmacological activity. Although the method developed here is sensitive and rapid enough to quantify silodosin in human plasma, it does not allow the analysis of KMD-3213G. The other two methods have essentially used the same approach to study the safety and pharmacokinetics of silodosin [7] and the effect of genetic polymorphisms on silodosin pharmacokinetics in healthy Chinese subjects [8].

Only two reports discuss simultaneous determination of silodosin and KMD-3213G in human plasma $[9,10]$ based on LC-MS/MS analysis. The method reported by Nair et al. [9] was developed and validated in spiked plasma samples using solid-phase extraction. Based on the optimized conditions, the recovery of silodosin was close to $60 \%$ and that of KMD-3213G around 90\%. The linear concentration range for silodosin and KMD-3213G was $0.502-207.376$ and $4.121-302.836 \mathrm{ng} / \mathrm{ml}$, respectively. However, the method was presented with no discussion on method development and application thereof. In one very recent research study, Shah et al. [10] critically evaluated each step of analysis (e.g., including extraction and chromatography) that directly impacts the method performance for the quantification of silodosin and KMD-3213G. The important feature of the method was complete baseline separation of the analytes and the selectivity achieved in lipemic and hemolyzed plasma samples, which are critical for reliable estimations in plasma samples. Though the analysis time was 6 min on Symmetry C18 column under gradient elution, the method was fivefold-times more sensitive $(0.10 \mathrm{ng} / \mathrm{ml})$ compared with all other methods. Moreover, the method required just $300 \mu \mathrm{l}$ plasma sample for processing. Further, the method was successfully applied for a bioequivalence study in healthy subjects and the reliability in the measurement of subject samples was demonstrated by reanalysis of selected incurred samples.

Although this area holds great promise for fine-tuning therapy and reducing the side effects of nonsubtype $\alpha_{1}$-AR antagonists, bioanalytical developments for silodosin seem to be minuscule since its approval. Thus, there is tremendous scope for the development of novel LC-MS/MS-based methods that integrate the following aspects:

- Analysis of the inactive metabolite, KMD-3293 along with the parent drug and its active glucuronide metabolite;

- Simultaneous analysis for a possible combination therapy involving tamsulosin or dutasteride with silodosin for $\mathrm{BPH}$-associated LUTS;

- Implementation of design of experiment (DOE) approach for method development;

- Use of high-resolution MS (HRMS) for high-throughput analysis and automation.

The measurement of plasma concentration of drugs and its major active and inactive metabolites is of immense interest in various types of investigations, including possible drug-drug interactions, pharmacokinetic studies, associated side effects and toxicity data of metabolites. According to Watson Pharmaceuticals NDA document [11], the combined systemic exposure of both the metabolites was $60-90 \%$ higher than the parent drug in a pharmacokinetic study. This suggests the importance of evaluating the plasma concentration of the inactive silodosin metabolite, KMD-3293. Glucuronidation is always a major metabolic detoxification pathway, which results in the formation of inactive water-soluble species or metabolites that are more active than the parent drug. The bioanalytical assay should be adequately robust under different conditions to generate reliable results. Further, interconversion of the drug-metabolite pair is also important at every step of the assay. During mass detection, glucuronide fragmentation should always be evaluated to avoid overestimation of the parent drug concentration. Additionally, the chromatographic separation of the metabolite from the parent drug is an equally important aspect of method development.

The other facet that could be explored is the suitability of the method to analyze fixed dose combination formulations, as silodosin monotherapy associated with a minor adverse event such as ejaculation failure in some cases [12]. The assessment of combination therapy comprising of silodosin with other uroselective drugs that have a complimentary mode of action can help to prevent such adverse events. This will further push the development of fixed-dose combination therapy in the market and allow for better compliance with minimal side effects. Furthermore, to develop an efficient bioanalytical methodology, the DOE approach is essential to prove method robustness. Traditional method development is realized by optimizing one parameter at a time 
(e.g., mobile composition, $\mathrm{pH}$, temperature etc.), while DOE can assist to account for the most probable interactions between various factors that affect the bioanalytical procedure. This leads to precise estimations with minimum experimental workup. To find the most influential operating parameter, different DOE methods, such as BoxBehnken design, Fractional Factorial design, Central Composite design and Plackett-Burman design, can be screened. For the selection of the optimal values of important bioanalytical parameters, response surface designs can then be evaluated $[13,14]$.

Over the past two decades, LC-MS/MS using triple quadrupole mass spectrometers has evolved into a mainstream approach for the quantitative bioanalysis of drugs and metabolites. In a routine sense, the determination of drugs in biological samples is considered as a multistep procedure involving calibration, sample pretreatment, chromatographic separation and detection by MS. The new generation of high-resolution mass spectrometry systems offer high-throughput quantitative analyses in combination with information-rich qualitative data with high sensitivity, scan-to-scan reproducibility, accuracy, dynamic range and resolution. This approach can lead to short analytical run times (typically $0.5-1.5 \mathrm{~min}$ ) for targeted quantitative analysis of silodosin, its metabolites and other uroselective agents in a fixed-dose combination therapy. With the ever-growing role of automation in bioanalytical approaches, especially in the area of sample processing and online tandem chromatographic techniques (such as multidimensional development or ultra-performance convergence chromatography), there can be considerable saving in operation time with increased separation selectivity. This can further enhance the productivity of the method with superior results.

\section{Conclusion}

So far, LC-MS/MS has been the indispensible tool for quantitative bioanalysis of silodosin, a promising candidate to treat BPH-associated LUTS. New advanced bioanalytical methods with higher sensitivity, speed, selectivity and robustness are required to increase the domain of analysis for silodosin and its metabolites. With the existing LC-MS/MS methods, it is difficult to unravel new tasks of dealing with combination therapy. More novel and fit-for-purpose bioanalytical methods are essential to meet this requirement. As it stands today, bioanalytical developments for silodosin and its metabolites are at a minuscule level, thus, there lies a tremendous scope for further development of novel bioanalytical platform using LC-MS/MS to extend the domain of analysis.

Financial \& competing interests disclosure

The authors have no relevant affiliations or financial involvement with any organization or entity with a financial interest in or financial conflict with the subject matter or materials discussed in the manuscript. This includes employment, consultancies, honoraria, stock ownership or options, expert testimony, grants or patents received or pending, or royalties.

No writing assistance was utilized in the production of this manuscript.

\section{References}

1. Schauer IG, Rowley DR. The functional role of reactive stroma in benign prostatic hyperplasia. Differentiation 82(4-5), 200-210 (2011).

2. Kawabe K, Yoshida M, Homma Y. Silodosin, a new $\alpha_{1 \mathrm{~A}}$-adrenoceptor-selective antagonist for treating benign prostatic hyperplasia: results of a Phase III randomized, placebo-controlled, double-blind study in Japanese men. BJU Int. 98(5), 1019-1024 (2006).

3. Yamanishi T, Mizuno T, Tatsumiya K, Watanabe M, Kamai T, Yoshida K. Urodynamic effects of silodosin, a new alpha 1A-adrenoceptor selective antagonist, for the treatment of benign prostatic hyperplasia. Neurourol. Urodyn. 29(4), 558-562 (2010).

4. Yamanishi T, Kaga K, Fuse M, Shibata C, Kamai T, Uchiyama T. A six year follow up of silodosin monotherapy for the treatment of LUTS/BPH: what are the factors for continuation or withdrawal? Int. J. Urol. 22(12), 1143-1148 (2015).

5. Cantrell MA, Bream-Rouwenhorst HR, Hemerson P, Magera JS Jr. New drug approvals: silodosin for benign prostatic hyperplasia. Ann. Pharmacother. 44(2), 302-310 (2010).

6. Zhao X, Liu Y, Xu J et al. Determination of silodosin in human plasma by liquid chromatography-tandem mass spectrometry. J. Chromatogr. B 877(29), 3724-3728 (2009).

7. Zhou Y, Sun PH, Liu YW, Zhao X, Meng L, Cui YM. Safety and pharmacokinetic studies of silodosin, a new $\alpha 1 A$-adrenoceptor selective antagonist, in healthy Chinese male subjects. Biol. Pharm. Bull. 34(8), 1240-1245 (2011).

8. Wang Z, Xiang Q, Cui Y, Zhao X, Zhou Y. The influence of UGT2B7, UGT1A8, MDR1, ALDH, ADH, CYP3A4 and CYP3A5 genetic polymorphisms on the pharmacokinetics of silodosin in healthy chinese volunteers. Drug Metab. Pharmacokinet. 28(3), 239-243 (2013).

9. Nair SM, Kumar R, Sharma M, Karia D. Development and validation of high performance LCMS methods for estimation of silodosin and silodosin $\beta$-D-glucuronide in human plasma. Pharm. Anal. Chem. 2(3), 1-6 (2016). 


\section{Editorial Shah \& Shrivastav}

10. Shah PA, Shrivastav PS. Determination of silodosin and its active glucuronide metabolite, KMD-3213G in human plasma by LC-MS/MS for a bioequivalence study. Biomed. Chromatogr. doi:10.1002/bmc.4041 (2017) (Epub ahead of print).

11. Rapaflo ${ }^{\circledR}$ (silodosin), package insert. Centre for Drug Evaluation and Research. NDA 22-206, Watson Pharmaceuticals Inc., CA, USA; October 2008.

12. Montorsi F, Gandaglia G, Chapple C, Cruz F, Desgrandchamps F, Llorente C. Effectiveness and safety of silodosin in the treatment of lower urinary tract symptoms in patients with beningn prostrate hyperplasia: a European Phase IV clinical study (SiRE study). Int. J. Urol. 23(7), 572-579 (2016).

13. Vander Heyden Y, Nijhuis A, Smeyers-Verbeke J, Vandeginste BG, Massart DL. Guidance for robustness/ruggedness tests in method validation. J. Pharm. Biomed. Anal. 24(5), 723-753 (2001).

14. Cochran WG, Cox GM. Some methods for the study of response surfaces. In: Experimental Designs (2nd Edition). Martens H, Martens M (Eds). Wiley, NJ, USA (2001). 